\section{IV\&V Assurance Case Design for Artemis II}

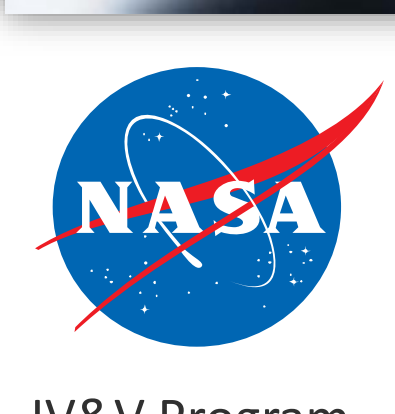

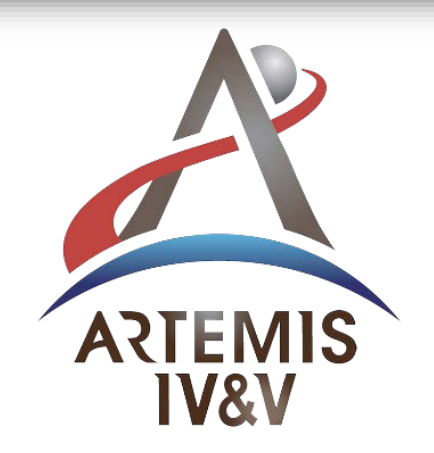

Gerek Whitman

SAIC.

Paul Amoroso

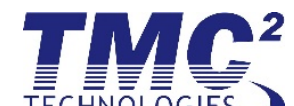

TECHNŌLLOGIES

Greg Black

SAIC.

Deneen Marculaitis-Granger

5AIC.

Justin Smith

NASA Independent

Verification \& Validation

John Bradbury

SAIC.

Wes Deadrick

NASA Independent

Verification \& Validation 


\section{NASA's IV\&V Program}

- NASA's Independent Verification \& Validation (IV\&V) Program reports to the Office of Safety and Mission Assurance (OSMA)

- Technically, Managerially, and Financially Independent

- Located in Fairmont, West Virginia

- NASA IV\&V employs systems engineering processes and rigorous methodologies for evaluating the correctness and quality of software products on NASA's highest profile missions

- Full Lifecycle

- Mission Oriented

- Capability Based

- NASA IV\&V goal: Add evidence-based assurance that minimizes the overall risk of NASA mission software

- In Phase

- Product Focused

- Risk Driven

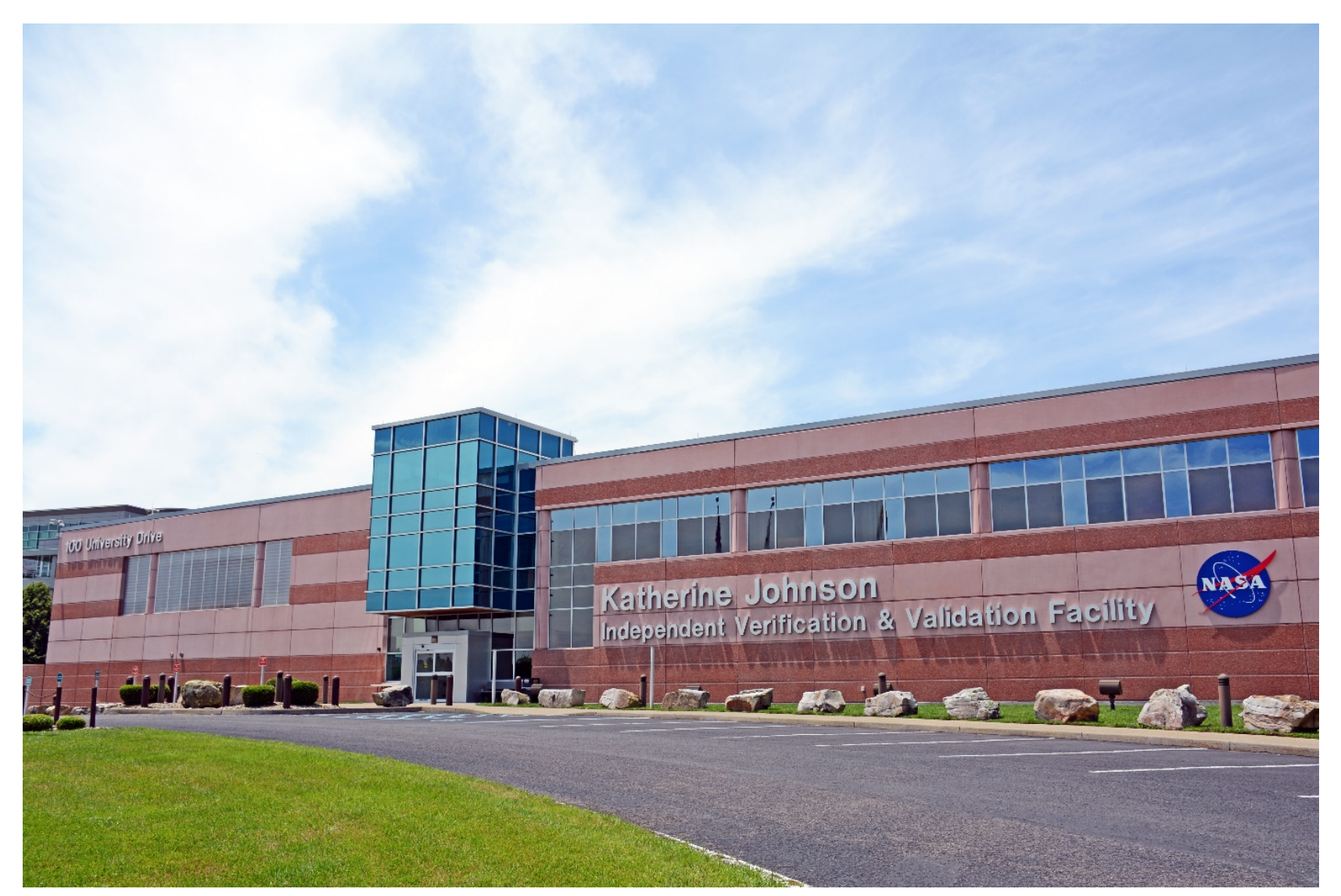

V\&V Assurance Case Design for Artemis II 


\section{NASA's Artemis Program}

\section{Artemis Phase 1: Path to The Lunar Surface}

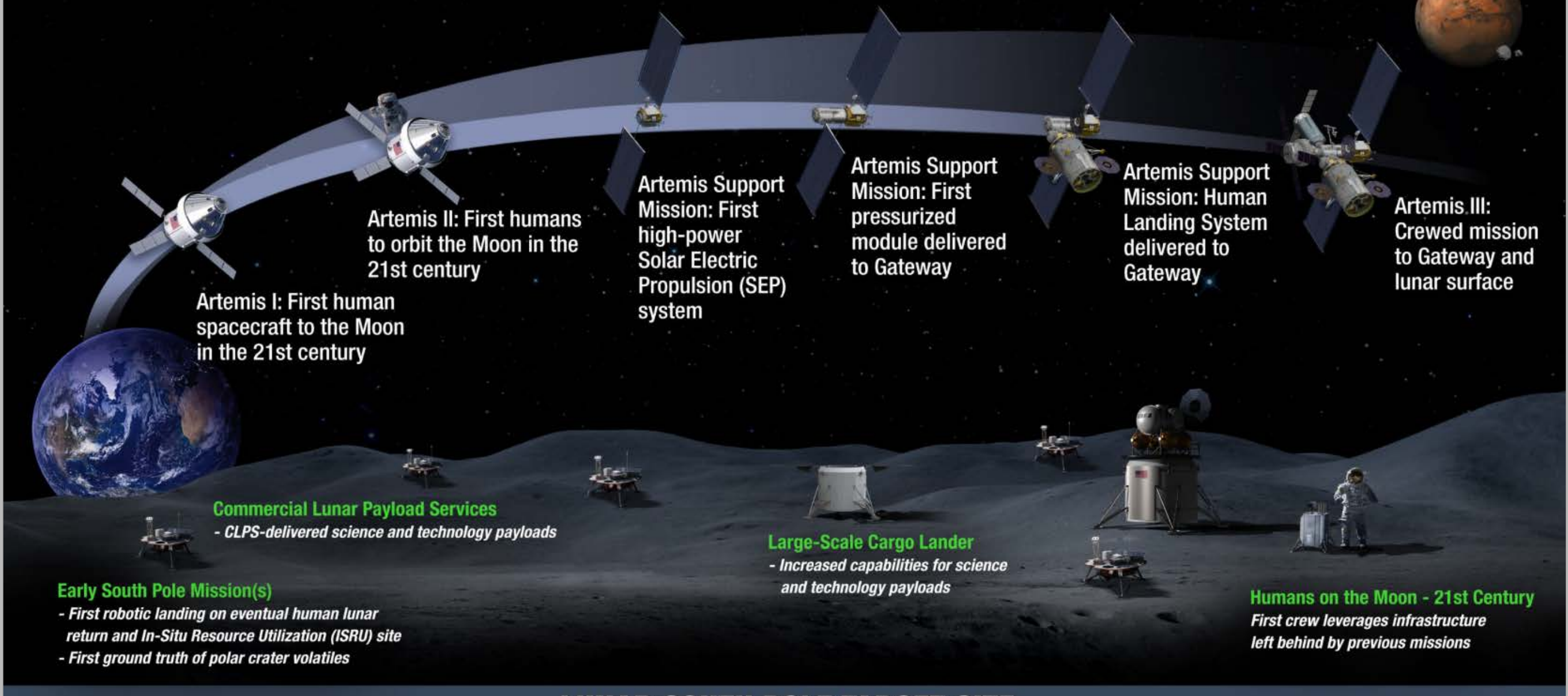

LUNAR SOUTH POLE TARGET SITE 


\section{Artemis IV\&V}
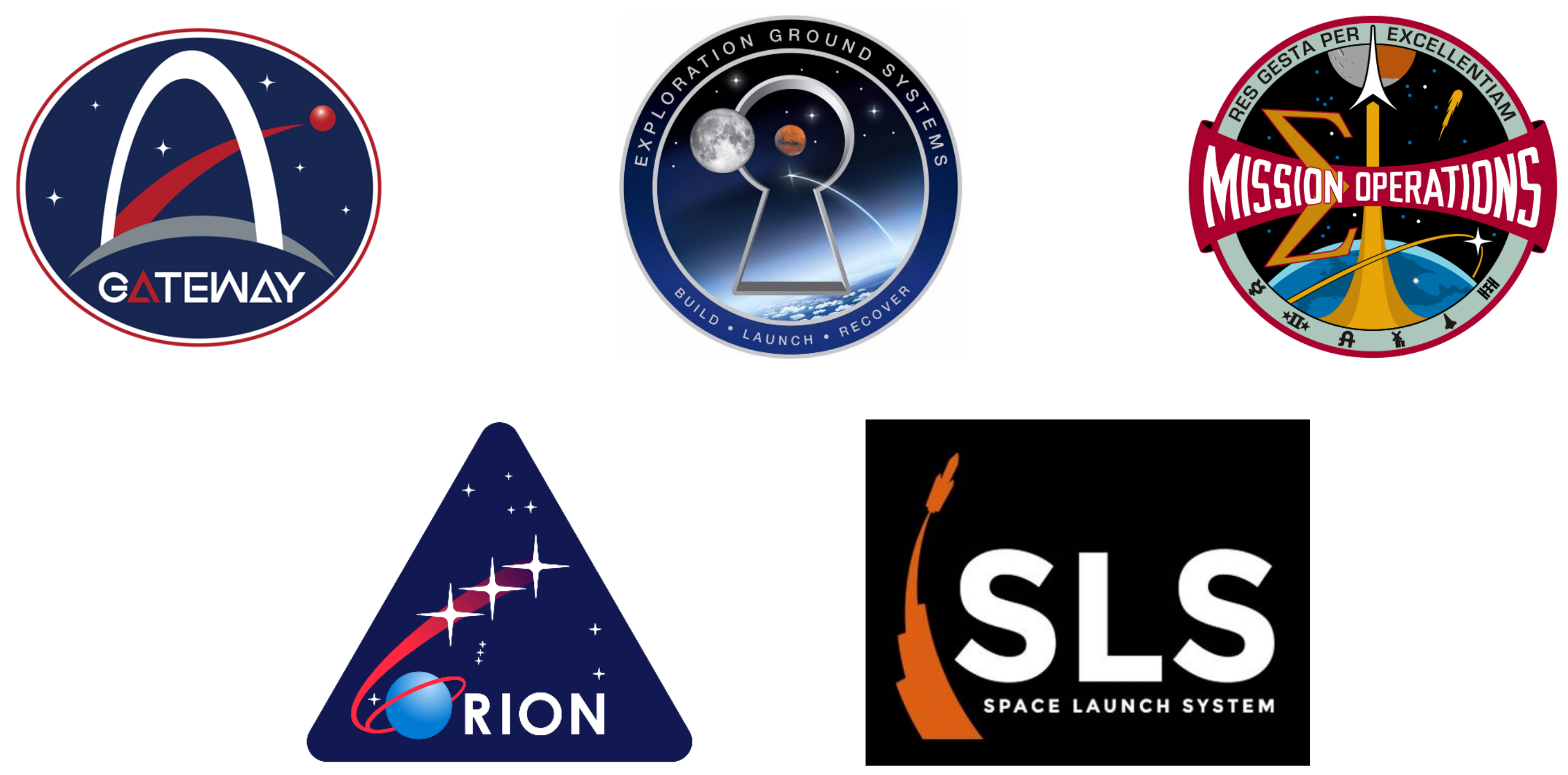


\section{Challenges}

- NASA IV\&V executes analysis on software artifacts, but in order to communicate effectively with stakeholders, we make assurance statements about mission capabilities

- Differences in planning and execution between the individual IV\&V project teams introduced challenges

- Risk assessment and prioritization approach was only loosely shared; analysis target priorities did not always line up across teams

- Aggregation of assurance conclusions did not always result in a cohesive message across project teams

- Gaps in assurance, particularly with respect to system and software integration, were difficult to identify and address

- Assurance data is difficult to objectively quantify, and our tooling approach made it difficult to manage, track, and keep our qualitative data up-to-date 


\section{Artemis II Mission Overview}

\section{ARTEMIS II}

Crewed Hybrid Free Return Trajectory, demonstrating crewed flight and spacecraft systems performance beyond Low Earth Orbit (LEO)

(1) LAUNCH 2 ENTER EARTH ORBIT
Perigee Raise Maneuver
(PRM) by Interim

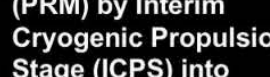
Stage (IICS) into
$100 \times 1545 \mathrm{nmi}$

orbit

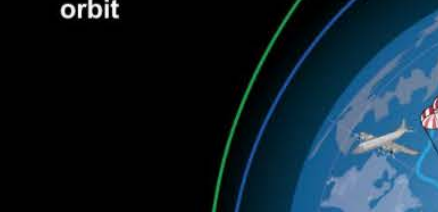

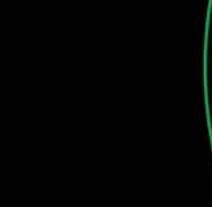

\section{APOGEE
RAISE
BURN}

BURN
TO HIGH

EARTH ORBIT
Followed by IC Followed by ICPS
separation and Orion systems checkout

(4) ORION TRANS-LUNAR INJECTION (TLI) BY ORION'S MAIN ENGINE

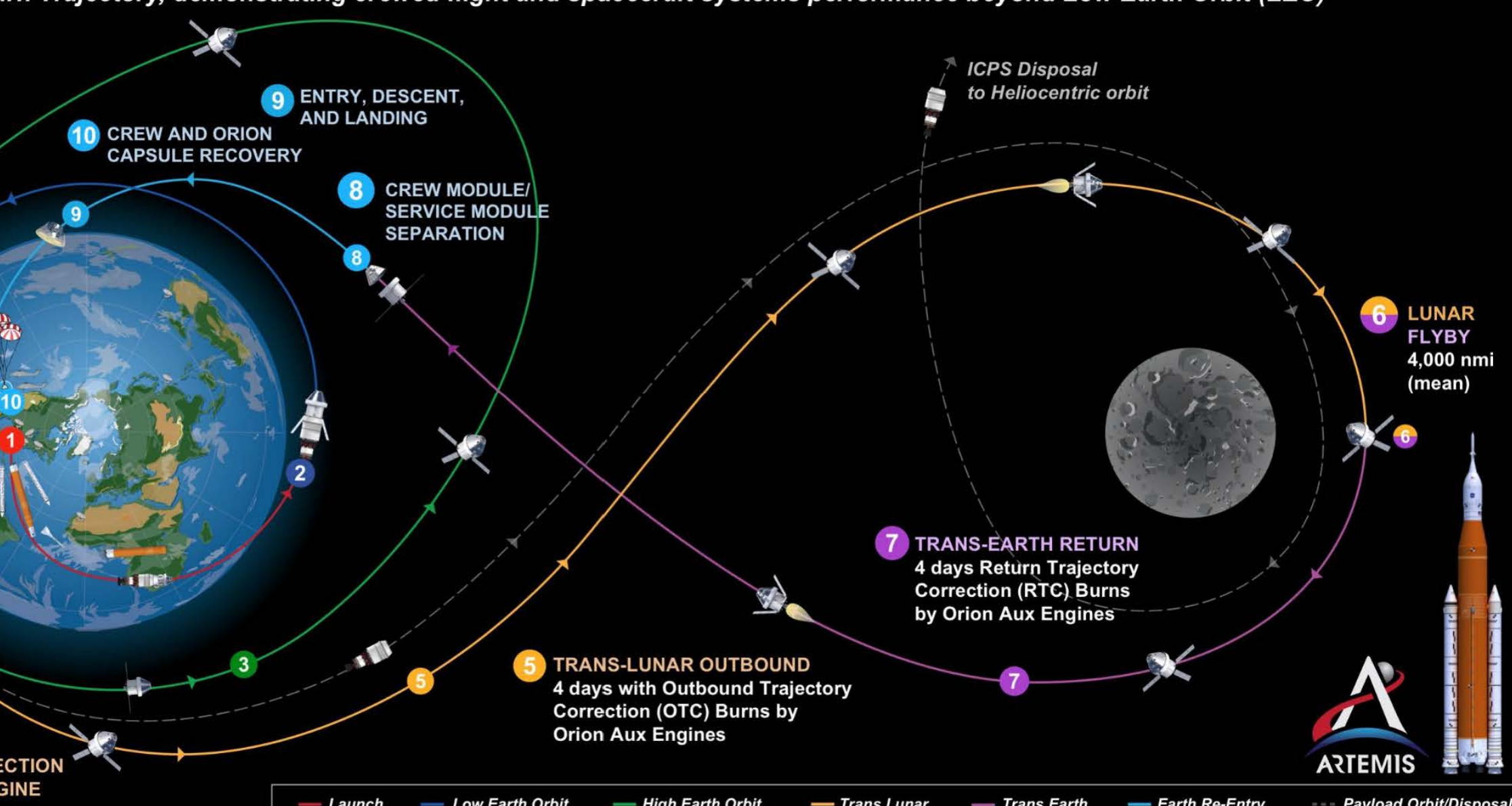

SLS Configuration (Block 1) with Human Rated ICPS | $15 \times 1200 \mathrm{nmi}(27.8 \times 2222.4 \mathrm{~km})$ insertion orbit | 28.5 deg inclination 4 astronauts | Mission duration: 10 Days | Re-entry speed: 24,500 mph (Mach 32) 


\section{Solutioning}

- The IV\&V project teams gathered for a Process Development Kaizen (PDK) Lean event

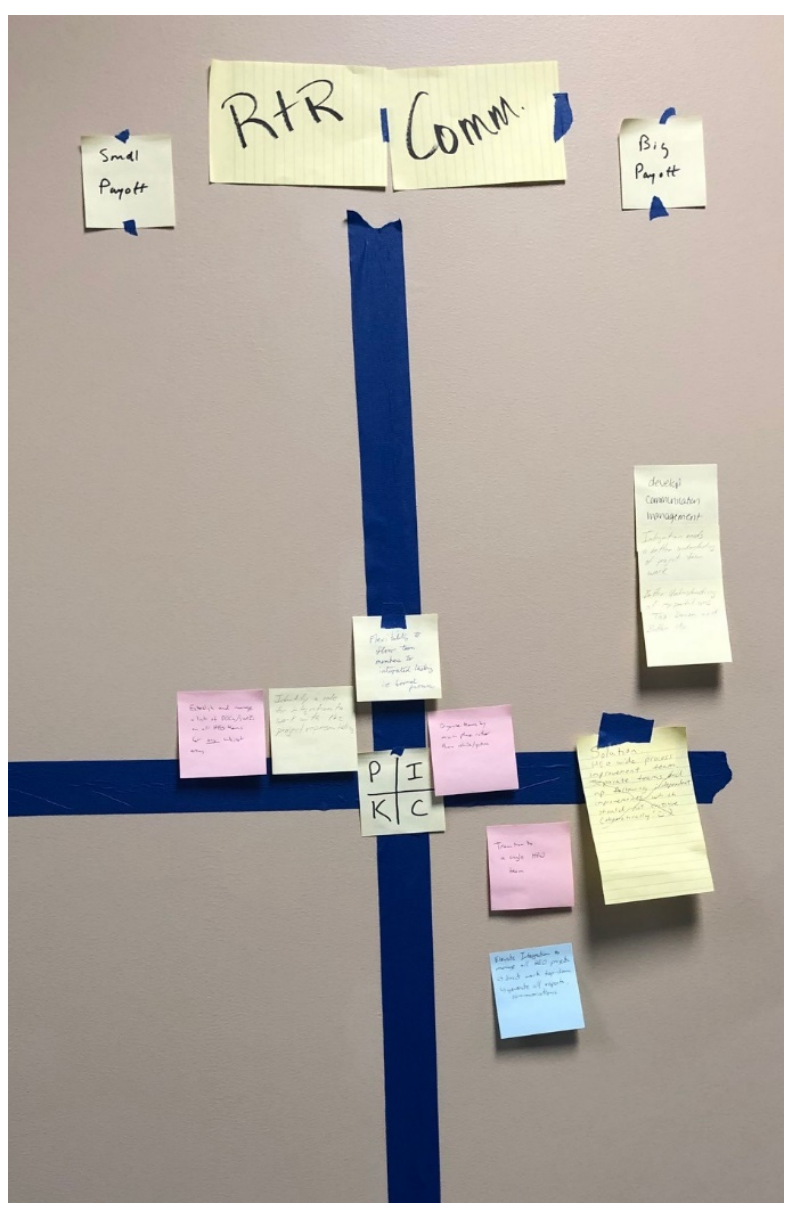

- Problem Statement: Our Artemis IV\&V projects operate as independent projects. The projects' outputs are varied in some form and make it difficult to communicate across projects, roll-up assurance to the mission level, and prioritize work and resources across projects. This approach results in numerous process inefficiencies and variation in the deliverables with Artemis IV\&V.

- Primary Objective: For Artemis II and beyond, determine an Artemis-wide workflow to support scoping, performing, capturing and reporting assurance analysis independent of mission.

- Primary output was an Implementation Plan identifying three further process development initiatives, as well as further requests from IV\&V leadership

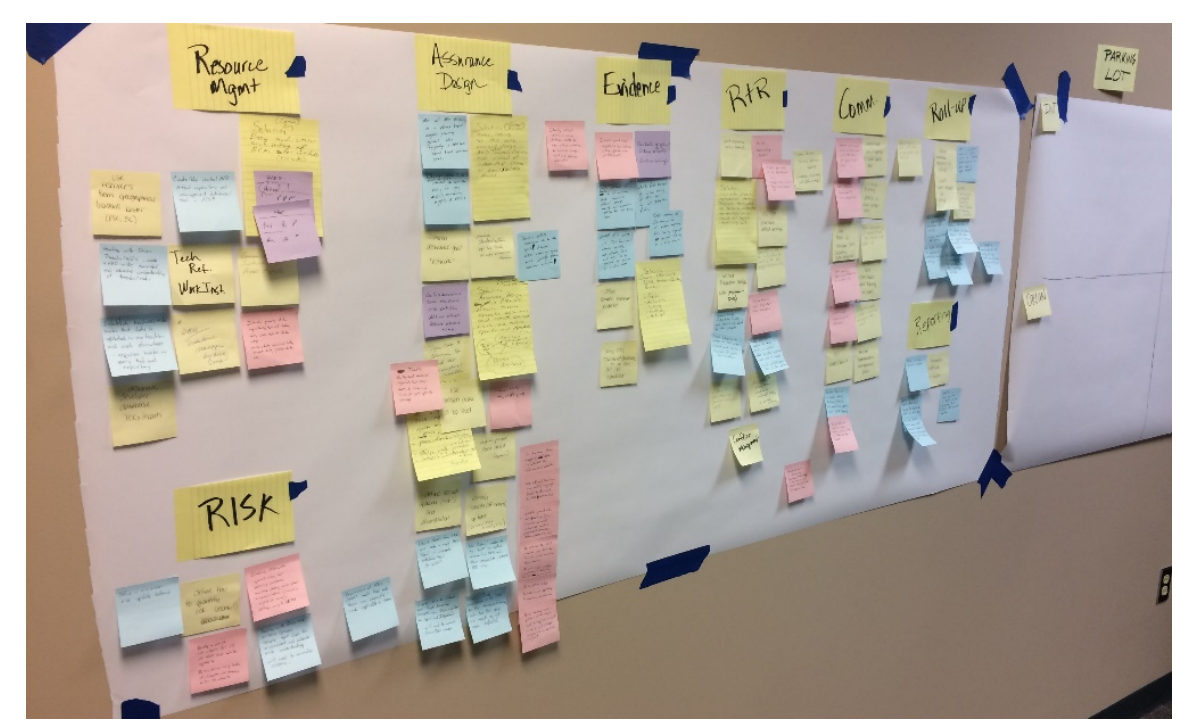




\section{Assurance Cases}

- An assurance case is a structured argument

- The GSN syntax provides a graphical notation for documenting an assurance case that supports scalability

- IV\&V realized that we can build an assurance case that captures:

- The decomposition of capabilities down to software components/functions

- The strategy IV\&V employs in planning analysis

- The evidence captured as a result of analysis

- We still needed a way to build in a risk assessment layer over top of the assurance case to determine which

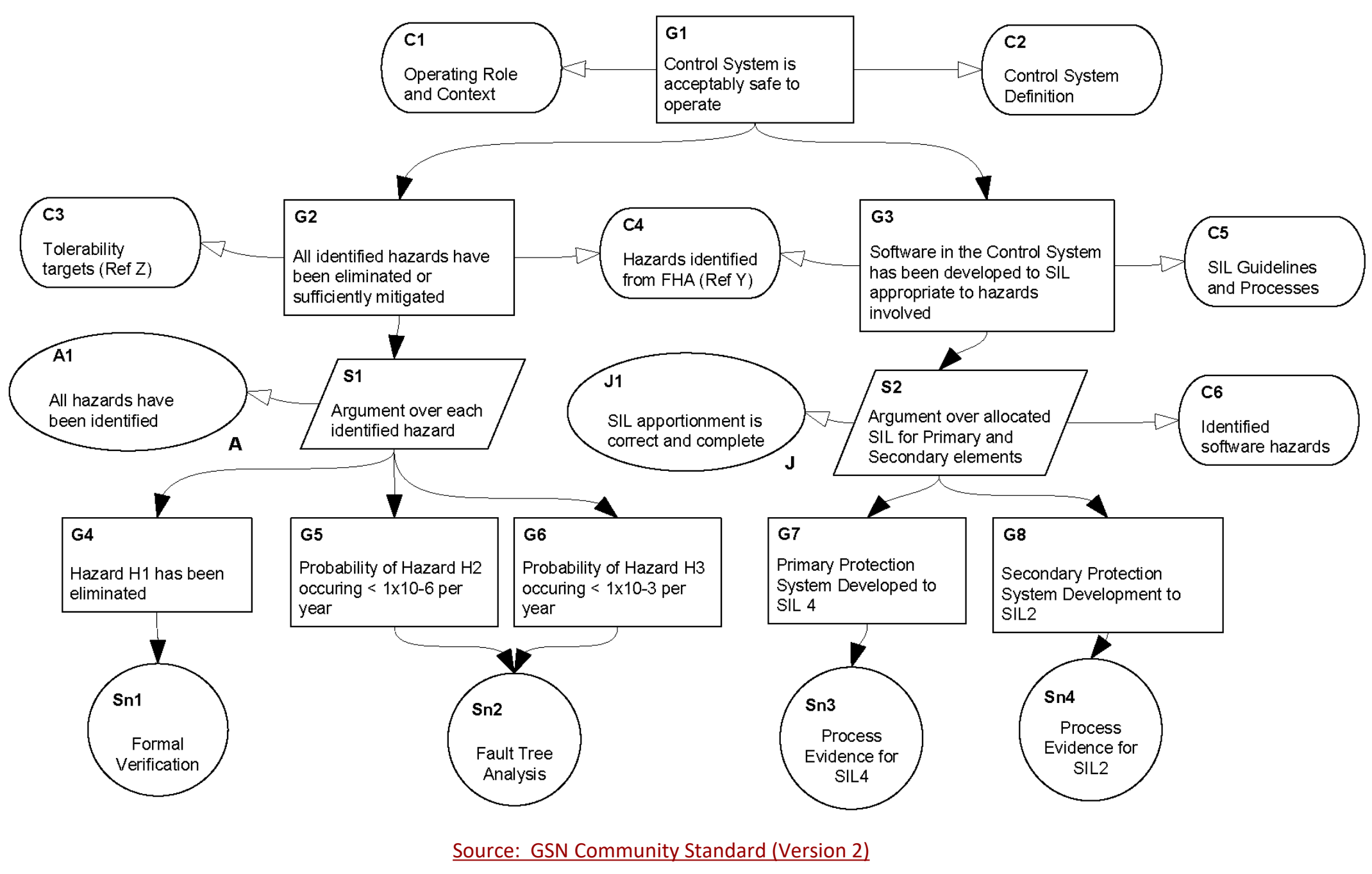
branches should be in focus 


\section{Implementation}

\section{Assurance Architecture Focus Group}

Objective: Develop the process and determine the tooling by which Artemis IV \&V will develop and maintain the Assurance Case

Additional Goals:

- Lay out the first few levels of claims in the Artemis Assurance Case

- Map existing assurance architecture from Artemis I into the new Assurance Case

- Identify training needs

\section{Risk Assessment Team}

Objective: Develop the process and criteria by which all Artemis IV\&V teams will conduct risk assessments for prioritizing at each level of decomposition

Additional Goals:

- Support selection of risk criteria and scoring with relevant industry research

- Validate the choice of criteria with a scoping/prioritization exercise based on Artemis I data

- Define an appropriate cadence to revisit risk assessments

IV\&V Program
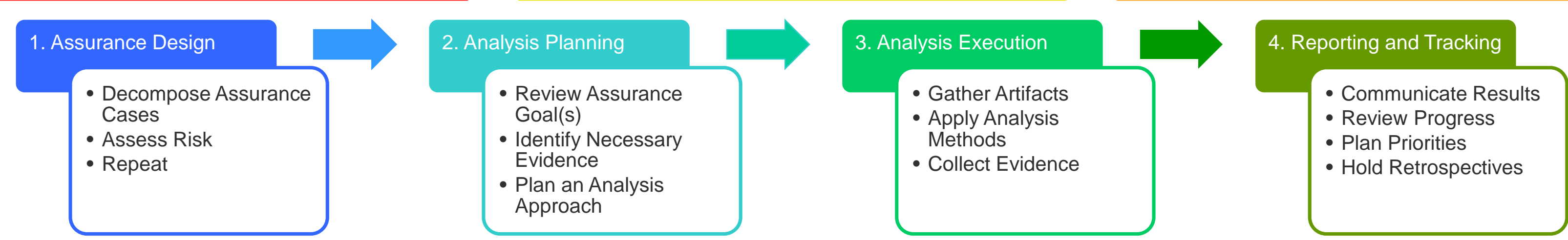


\section{The Experience Thus Far}

Benefits

- The Lean event had the immediate effect of increasing cross-team communication, which continues almost a year later

- Establishing an Artemis IV\&V Program has begun synchronizing planning and execution across all of the project teams

- The Artemis assurance case has made it easier to see the integration points where additional risk could exist

- Analysts have found building the assurance case to be an intuitive activity that helps build their system understanding
Drawbacks

- New training necessary to teach analysts how to build and use the assurance case

- A single tool solution that supports our process and requirements (esp. for multiuser platform) does not yet exist

- Growing pains across the Artemis team as analysts adapt to these new methods of planning and executing their work

- Building the assurance case requires additional effort to formally represent the assurance design 


\section{Looking Ahead}

- Stepping up adoption and use of the Artemis assurance case from $\sim 10$ analysts (currently) to the whole Artemis IV\&V team ( 85 analysts)

- Integrating the assurance case with our other tools

- Simultaneous development of assurance cases for Artemis II and III

- Migrating evidence and assurance from previous Artemis I assurance design constructs into the Artemis assurance case

- Building external communication and reporting features using the assurance case and toolchain

- Maintaining continuous improvement activities across the entire Artemis team 


\section{Questions?}




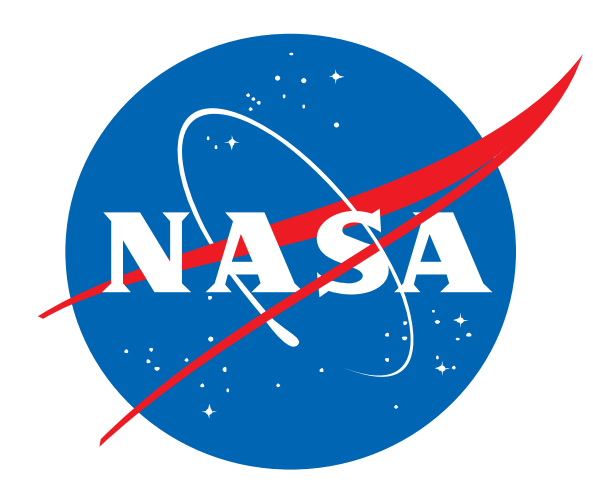

